\title{
Nach genetischen Ursachen fahnden
}

Fragestellung: Anhand eines großen genomischen Datensatzes aus mehreren genomweiten Assoziationsuntersuchungen (GWAS) wurde überprüft, ob eine interstitielle Deletion auf Chromosom 22q11.2 einen Risikofaktor für die ParkinsonKrankheit darstellt.

Hintergrund: Die Parkinson-Krankheit ist genetisch heterogen. Immer mehr Fälle der Erkrankung werden auf spezifische Genmutationen zurückgeführt. Kasuistisch wurde in einer Arbeit von 2013 [1] anhand von acht Fällen eine Assoziation zwischen dem sogenannten 22q11.2-Deletions-Syndrom und der Parkinson-Krankheit beschrieben. Diese Deletion, die meist eine Größe von etwa drei Megabasen aufweist, ist mit einer Häufigkeit von 25 auf 100.000 Lebendgeburten die häufigste angeborene interstitielle Deletion des Menschen, und wurde bislang mit einer Reihe von Fehlbildungen und Erkrankungen, wie kraniofazialen Dysmorphien, Herzfehlern und auch der Schizo-

Mok KY, Sheerin U, Simon-Sanchez J et al. Deletions at 22q11.2 in idiopathic Parkinson's disease: a combined analysis of genomewide association data. Lancet Neurol 2016; 15: 585-96 phrenie in Verbindung gebracht.

Patienten und Methodik: Mok et al. untersuchten die Häufigkeit der 22.q11.2-De- letion bei 9.387 Patienten mit Parkinson-Syndrom und 13.863 Kontrollpersonen, wofür sie genomische Datensätze verwendeten, die aus insgesamt vier genomweiten Assoziationsuntersuchungen (GWAS) stammten.

Ergebnisse: Eine 22q11.2-Deletion wurde bei insgesamt acht $\mathrm{Pa}$ tienten $(0,089 \%)$, aber bei keiner Kontrollperson gefunden $(\mathrm{p}=0,0082)$. Sechs der Patienten mit Deletion hatten einen frühen Erkrankungsbeginn ( $<45$ Jahre), was immerhin 0,49\% der Patienten dieser Subgruppe entspricht. Keiner der deletionstragenden Patienten war durch typische Zeichen eines 22q11.2-Deletions-Syndroms aufgefallen, mehrere Patienten hatten jedoch klinische Auffälligkeiten wie eine Lippen-Kiefer-Gaumenspalte, Herzklappenanomalien oder psychiatrische Auffälligkeiten vor Beginn der Parkinson-Krankheit, die als „form fruste“ dieses Syndroms aufgefasst werden können. Bis auf den auffällig frühen Krankheitsbeginn entsprach das neurologische Syndrom dem typischen idiopathischen Parkinson-Syndrom.

Schlussfolgerungen: Deletionen eines drei Megabasen großen Abschnitts auf Chromosom 22q11.2 wurden als eine weitere genetische Ursache der Parkinson-Krankheit gefunden. Bei Patienten mit früh beginnendem Parkinson-Syndrom sollte nach möglichen genetischen Ursachen gefahndet werden.

\section{- Kommentar von Thomas Gasser, Tübingen}

\section{Die Aufklärung der Ursache kann praktische Konsequenzen haben}

Die Arbeit fügt der wachsenden Liste von seltenen erblich bedingten Formen der Parkinson-Krankheit eine weitere hinzu. Obwohl jede einzelne dieser Formen selten oder sogar sehr selten ist, machen sie in ihrer Gesamtheit doch einen nicht unerheblichen, und vor allem zunehmenden Teil aller Erkrankungsfälle aus. Insbesondere bei frühem Erkrankungsbeginn (vor dem 45. Lebensjahr) und bei nicht klar einzuordnenden zusätzlichen klinischen Auffälligkeiten muss daran gedacht werden. Die Aufklärung einer genetischen Ursache kann praktische Konsequenzen für die genetische Beratung des Patienten und seiner Angehörigen aber auch für das Management der Erkrankung selbst haben, was zum Beispiel bei der 22q11.2Deletion die Suche nach begleitenden therapiewürdigen kardialen Anomalien beinhaltet. Bei manchen anderen genetischen Parkinson-Syndromen, zum Beispiel der durch Mutationen im Gen für die Glucocerebrosidase (GBA) mitbedingten Erkrankung, sind auch schon erste mutationsspezifische Therapiestudien in Vorbereitung [2].

\footnotetext{
Referenzen:

1. Butcher NJ et al. JAMA Neurology 2013; 70: 1359-66

2. Schapira AH et al. Mov Disord 2016; 31: 830-5
} 